\title{
COMUNICACIÓN
}

\section{Contaminación de aceras con enteroparásitos caninos en Corrientes, Argentina}

\author{
ALICIA M. F. MILANO* y ELENA B. OSCHEROV*
}

\section{SIDEWALKS CONTAMINATION WITH CANINES ENTEROPARASITES IN CORRIENTES, ARGENTINE}

The present investigation was carried out in order to evaluate the canine parasites contamination in Corrientes city sidewalks. The samples of canine faecal matter proceeding the city were analysed. Samples were processed individually by the following methods: sedimentation through centrifugation and Willis flotation. From 362 samples analized, 58.6\% $(n=212)$ were positive. The species identified were Ancylostoma sp. (41.2\%), Toxocara canis (16.0\%), coccidios (10.5\%), Giardia sp. (5.5\%), Trichuris vulpis (4.7\%), Diphyllobothriidae (2.8\%) and Dipylidium caninum (0.3\%). It is stated once again the necessity to stablish rules and strict sanitary and environmental controls.

Key words: Zoonosis, Urban contamination, Canine parasites, Sidewalk.

\section{INTRODUCCIÓN}

Algunos enteroparásitos caninos, son capaces de infectar al hombre. La materia fecal es uno de los elementos a través del cual los parásitos se diseminan al tomar contacto con el medio externo. Asimismo el contacto del hombre con las heces o fomites (tierra, alimentos, agua) favorece la transmisión feco-oral y dérmica.

Las parasitosis del perro que más importancia tienen en salud pública son las producidas por ancylostomídeos, Toxocara canis, y Echinococcus granulosus $^{1-3}$. Si bien estas infecciones no son necesariamente causa de muerte, tienen alta morbilidad dado que interactúan con otros factores y se ubican entre las enfermedades de mayor importancia económica y como zoonosis ${ }^{4}$.

En Argentina se detectó la presencia de formas infectantes de las especies parasitarias antes mencionadas en sitios públicos urbanos tales como paseos, plazas, balnearios y aceras ${ }^{5-9}$.

Trabajos de investigación llevados a cabo en la ciudad de Corrientes demuestran la presencia de formas parasitarias en heces de perros encontradas en paseos y balnearios públicos ${ }^{6,7}$. La contaminación de esos espacios se produce por perros vagabundos y con dueños, ya que tienen fácil acceso a la vía pública. Por eso es que en el ámbito urbano, es frecuente la presencia de heces diseminadas en las aceras. Estos espacios no solo son vías de tránsito sino que conforman extensionesen donde juegan los niños; por lo tanto la población infantil es uno de los grupos mas expuestos al foco de transmisión.

El presente estudio se realizó con el objeto de evaluar la contaminación de las aceras con parásitos caninos en la ciudad de Corrientes.

* Cátedra de Parasitología. Departamento de Biología. Facultad de Ciencias Exactas y Naturales y Agrimensura. Universidad Nacional del Nordeste. Av. Libertad 5470.3400. Corrientes. Argentina. E- mail: milano@exa.unne.edu.ar 


\section{MATERIAL Y MÉTODOS}

Área de estudio: La ciudad de Corrientes está localizada a $27^{\circ} 25^{\prime} \mathrm{LS}$ y $58^{\circ} 52^{\prime}$ LO. Existe una escasa variación anual entre las temperaturas estivales (media: $27^{\circ} \mathrm{C}$ ) e invernales (media: $16^{\circ}$ C) lo que determina un clima mesotermal y con un régimen pluvial de aproximadamente 1.500 $\mathrm{mm}$ anuales ${ }^{10}$. La mayor parte del área urbanizada está limitada por la margen izquierda del Río Paraná y la ruta Nacional $\mathrm{N}^{\circ} 12$.

Trabajo de campo: La investigación se llevó a cabo desde mayo de 2002 hasta mayo de 2003. Para su estudio, la ciudad fue dividida en nueve zonas (Tabla 1) utilizando las avenidas como límites entre cada una de ellas (Figura 1). En cada zona se seleccionaron al azar cinco manzanas. En cada una de las manzanas se realizó un censo de la materia fecal canina depositada en la acera. Se tomó una muestra de cada una de las heces observadas, las que se conservaron por separado en frascos rotulados, con formol al 10\%. Las heces se clasificaron según su consistencia en líquida, pastosa y seca.

Actividades de laboratorio: Cada muestra de materia fecal se procesó en forma individual mediante los métodos de sedimentación por centrifugación con agua y flotación de Willis (solución saturada de $\mathrm{NaCl}$ ). Se realizaron dos preparados de cada muestra, los que se colorearon con Solución Lugol y se observaron con microscopio óptico Olympus GH 30 (10x y 40x).

Para la identificación del género y especie de los parásitos se utilizó una clave específica ${ }^{11} \mathrm{y}$ las formas parasitarias encontradas se midieron y compararon con fotografías estándares. Se consideró positiva aquella zona que presentó al menos una muestra contaminada.

Análisis estadístico: Se aplicó la prueba de diferencia de proporciones (chi cuadrado) para comparar los métodos de procesamiento empleados y la prevalencia entre zonas.

\section{RESULTADOS}

El $100 \%$ de las zonas relevadas fue positivo. La prevalencia parasitaria general fue de 58,6\% $(\mathrm{n}=212 / 362)$. El número promedio de muestras de heces recolectado por cuadra fue 4,7 (mínimo 1 , máximo 20).

Se verificó mayor porcentaje de muestras positivas en las heces de consistencia pastosa $(45,3 \%)$. Asimismo se constató diferencia entre los métodos utilizados, observándose mayor recuperación de formas parasitarias con el método de sedimentación por centrifugación $\left(\mathrm{chi}^{2}\right.$ $=12,7 ; \mathrm{gl}=1 ; \mathrm{pd} \leq 0,0001$ ).

En la Tabla 1 se aprecian los valores de la prevalencia por zona y especie parasitaria. El género más frecuentes fue Ancylostoma sp. y, en contraposición, D. caninum se encontró en una sola de las muestras. El 2,8\% de las muestras presentó huevos de Diphyllobothriidae.

La mayor proporción de muestras positivas se encontró en la zona 9 mientras que la menor proporción se halló en la zona 1. En la zona que comprende el casco histórico de la ciudad (zona 1) se constató una frecuencia relativa de $26,3 \%$

Tabla 1. Parásitos identificados en materia fecal canina recolectada en aceras de la ciudad de Corrientes. Corrientes, Argentina. 2003

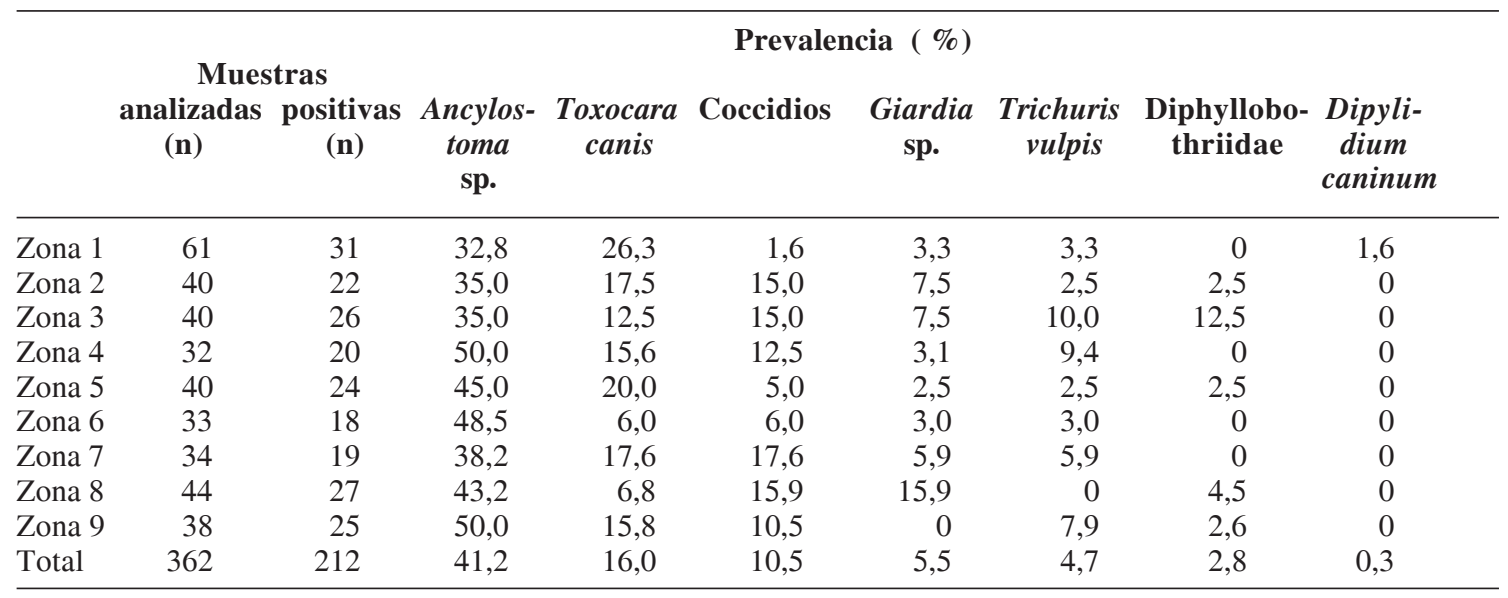




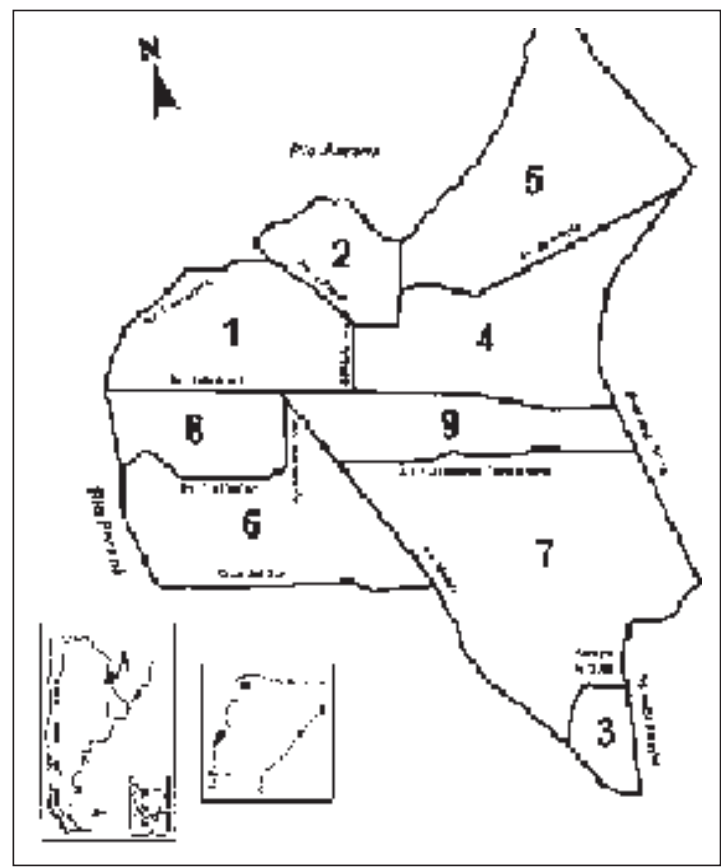

Figura 1. Zonas muestreadas de la ciudad de Corrientes.

para $T$. canis, mientras que en el resto de la ciudad esta especie se encontró representada entre el 6 y 20,0\%. Los huevos de Diphyllobothriidae se hallaron en mayor proporción $(12,5 \%)$ en las muestras de la zona más alejada del casco céntrico (zona 3). Ancylostoma sp., T. canis y coccidios se verificaron en todas las zonas. Entre los protozoos, se observó mayor frecuencia de coccidios que de Giardia sp.

Si bien hubo variación en los valores de prevalencia entre las zonas relevadas no se observaron diferencias significativas entre ellas $\left(\chi^{2}=3,92 ; \mathrm{gl}=8\right.$; pe $\left.\geq 0,86\right)$.

En el 30,2\% (64/212) de las muestras positivas se observó la presencia de más de una especie parasitaria. Las combinaciones biespecíficas más frecuentes fueron Ancylostoma sp. y T. canis; Ancylostoma sp. y coccidios; Ancylostoma sp. y Trichuris vulpis; en los hallazgos triespecíficos se observó Ancylostoma sp., Giardia sp. y coccidios; Ancylostoma sp., $T$. canis y T. vulpis; en sólo una de las muestras se observó la presencia simultánea de Ancylostoma sp., T. canis, Giardia sp. y coccidios.

\section{DISCUSIÓN}

Las especies halladas revisten importancia tanto para la salud canina como humana. Ancylostoma sp., T. canis y T. vulpis son los helmintos de mayor frecuencia. A pesar de la diferencias geográficas los resultados son similares a los obtenidos en Brasil ${ }^{12}$, Chile ${ }^{13}$ y distintas ciudades argentinas s, $^{5,9,9,14}$.

La contaminación de las heces con Ancylostoma sp. en las aceras fue muy alta, semejante a la citada para los paseos públicos de la ciudad de Corrientes $^{6}$, pero inferior a la constatada en los balnearios de la misma ciudad ${ }^{7}$ y en el peridomicilio de viviendas de dos localidades de Salta, Argentina ${ }^{14}$.

En plazas de la ciudad de La Plata se encontraron valores similares ${ }^{8}$. Sin embargo los valores correspondientes a muestras recolectadas en las aceras de esa ciudad son inferiores a los de la presente investigación; lo que podría atribuirse a diferencias metodológicas.

Respecto a $T$. canis la prevalencia obtenida supera a la hallada por otros autores ${ }^{6,7}$ pero coincide con la de otros ${ }^{14}$. La zona 1 (donde se encontró el valor mas alto para $T$. canis) corresponde al centro de la ciudad. En ella se pueden observar numerosas propiedades horizontales y los perros que habitan en los departamentos son llevados a defecar a las aceras al menos una vez por día. En la presente investigación no se pudo establecer la edad de los perros; no obstante, los valores encontrados se aproximan a la prevalencia de infección en cachorros menores a seis meses, la cual es cercana al $28,0 \%^{15}$; por lo tanto podría suponerse que los cachorros serían la principal fuente de contaminación de la acera en esta zona.

La frecuencia relativa de $D$. caninum probablemente esté subestimada, teniendo en cuenta que los proglótides sólo se encuentran en deposiciones frescas ${ }^{16}$.

Las zonas en las que se registró la presencia de huevos de Diphyllobothriidae (zonas 2, 3, 5, 8 y 9) están ubicadas en las cercanías de cuerpos de agua tales como desagüies pluviales abiertos, arroyos y el Río Paraná, lo que sugiere que el ciclo de transmisión está en relación con el agua. Sería necesario ampliar el conocimiento sobre identificación, la biología y ecología de este parásito a fin de evaluar su importancia epidemiológica en el área de estudio.

Además de los helmintos, se observó la presencia de protozoos; los más frecuentes fueron los coccidios. Esto coincide con estudios 
llevados a cabo en la ciudad de Mar del Plata, Buenos Aires ${ }^{5}$ y en dos ciudades de la provincia de Chubut ${ }^{17}$ en donde se constató la presencia de coccidios en heces recolectadas en plazas, aunque en menor cantidad.

Si bien sólo el 5,5\% de las muestras positivas presentó Giardia sp. no se debe minimizar su potencial zoonótico ya que diversos trabajos coinciden en la posibilidad de transmisión entre especies de mamíferos, incluyendo al hombre ${ }^{18}$.

En cuanto a la comparación entre zonas de la ciudad de Corrientes, se comprobó que no existen diferencias estadísticamente significativas, que lo demuestra cierta uniformidad en cuanto a los factores determinantes.

La acera es un ambiente poco propicio para el desarrollo de los geohelmintos, por lo tanto la potencialidad de transmisión de ancylostomídeos se vería reducida. Sin embargo los huevos de las otras especies de helmintos y los quistes de protozoos conservarían su potencial infectividad.

Los resultados de esta investigación reafirman la gravedad de la situación descripta en estudios previos y reflejan la necesidad de implementar medidas sanitarias más fuertes y controles más estrictos. Si se tiene en cuenta que muchas de las heces analizadas podrían ser de perros con dueño, el aspecto a reforzar sería la educación sanitaria, promoviendo la tenencia responsable de mascotas, la administración periódica de antiparasitarios y la recolección de materia fecal de la vía pública.

\section{RESUMEN}

Para evaluar la contaminación de las aceras con parásitos caninos en la ciudad de Corrientes, se tomó una muestra de cada una de 362 heces presentes en las aceras. Las muestras se procesaron en forma individual mediante los métodos de sedimentación por centrifugación con agua y flotación de Willis (solución saturada de $\mathrm{NaCl}$ ). La prevalencia parasitaria general fue de $58,6 \%(\mathrm{n}=212)$. Las especies identificadas fueron: Ancylostoma sp. (41,2\%), Toxocara canis (16,0\%), Coccidios (10,5\%), Giardia sp. $(5,5 \%)$, Trichuris vulpis (4,7\%), Diphyllobothriidae $(2,8 \%)$ y Dipylidium caninum $(0,3 \%)$.

\section{REFERENCIAS}

1.- ACHA P N, SZYFRES B. Zoonosis y enfermedades transmisibles comunes al hombre y los animales. Segunda
Edición. Pub Científica No 503. OPS 1992; 989 pp.

2.- ATÍAS A. Parasitología Médica. Pub Téc Mediterráneo Ltda. Chile 1998; 615 pp.

3.- BECK A. The ecology of stray dogs. A study of freeranging urban animals. York Press, Baltimore 1973.

4.- SCHANTZ P M. Toxocara larva migrans now. Am J Trop Med Hyg 1989; 41: 21-34.

5.- ANDRESIUK M V, DENEGRI G M, SARDELLA N H, HOLLMANN P. Encuesta coproparasitológico canina realizado en plazas públicas de la ciudad de Mar del Plata, Buenos Aires, Argentina. Parasitol Latinoam 2003; 58: 17-22.

6.- MARDER G, ULON S N, BOTTINELLI O R, et al. Infestación parasitaria de suelos y materia fecal de perros y gatos de la ciudad de Corrientes. Disponible en URL: http://www.unne.edu.ar/Web/cyt/cyt/2002/cyt.htm.

7.- MILANO A M F, OSCHEROV E B. Contaminación por parásitos caninos de importancia zoonótica en playas de la ciudad de Corrientes, Argentina. Parasitol Latinoam 2002; 57: 119-23.

8.- MINVIELLE M C, PEZZANI B C, BASUALDO FARJAT J A. Frecuencia de hallazgo de huevos de helmintos en materia fecal canina recolectada en lugares públicos de la ciudad de La Plata, Argentina. Bol Chil Parasitol 1993; 48: 63-5.

9.- ZUNINO M G, DE FRANCESCO M V, KURUC J A, et al. Contaminación por helmintos en espacios públicos de la provincia de Chubut, Argentina. Bol Chil Parasitol 2000; 55: 78-83.

10.- CARNEVALI R. Fitogeografía de la Provincia de Corrientes. Gobierno de la Provincia de Corrientes. Instituto Nacional Tecnología Agropecuaria. Corrientes, Argentina 1994; pp: 324.

11.- THIENPONT D, ROCHETTE F, VANPARIJS O F J. Diagnóstico de las helmintiasis por medio del examen coprológico. Johnson y Johnson de Argentina S.A. Comercial e Industrial. Div Veterinaria 1979; pp: 183.

12.- HOFFMANN A N, BELTRÃO N, AVILA BOTTON $\mathrm{S}$, et al. Intestinal nematodes of stray dogs as zoonoses agents in D. Pedrito city (RS Brazil). Bol Chil Parasitol 2000; 55: 92-3.

13.- CASTILLO D, PAREDES C, ZAÑARTU C, et al. Contaminación ambiental por huevos de Toxocara sp. en algunas plazas y parques públicos de Santiago de Chile, 1999. Bol Chil Parasitol 2000; 55: 86-91.

14.- TARANTO N J, PASSAMONTE L, MARINCONZ R, et al. Parasitosis zoonóticas transmitidas por perros en el Chaco Salteño. Medicina (Bs.Aires) 2000; 60: 217-20.

15.- VENTURINI L, RADMAN N E. Frecuencia de Toxocara canis, Ancylostoma caninum y Giardia sp. según el sexo y la edad, en caninos de La Plata (Bs. As., Argentina). Rev Med Vet (Bs As) 1988; 69: 161-5.

16.- GENNARI S M, KASAI N, PENA H F J, CORTEZ A. Ocorrência de protozoarios e helmintos em amostras de fezes de câes e gatos da cidade de São Paulo. Braz J Vet Anim Sci 1999; 36: 87-91.

17.- SÁNCHEZ P, RASO S, TORRECILLAS C, et al. Contaminación biológica con heces caninas y parásitos intestinales en espacios públicos urbanos en dos ciudades de la Provincia de Chubut. Patagonia Argentina. Parasitol Latinoam 2003; 58: 131-5.

18.- SCHANTZ P M. Parasitic zoonoses in perspective. Int J Parasitol 1991; 21: 161-70. 